\title{
Super-Graphene: The Role of Temperature on Radiation Resistance
}

Ondrej Dyck ${ }^{1}$, Jacob Swett ${ }^{2}$, Andrew Lupini ${ }^{1}$ and Stephen Jesse ${ }^{1}$

${ }^{1}$ Oak Ridge National Laboratory, Oak Ridge, Tennessee, United States, ${ }^{2}$ University of Oxford, NA, Alabama, United States

Graphene has been proposed to be useful for a wide range of devices. Interesting quantum properties emerge when the precise atomic structure can be specified [1,2]. Assembly of such precise structures is beyond the reach of current top-down fabrication techniques, however scanning transmission electron microscopy (STEM)-based methods may offer a path forward [3, 4]. The angstrom-scale electron probe enables some surprisingly precise atomic alterations such as bond inversions [5-7] and localized insertion of dopants [8-10]. To fully leverage these capabilities for patterning dopant and defect arrays, precise control of beam-induced atomic ejection ("knock-on" process) is desired.

A recent theoretical study has extended the description of the knock-on process in graphene to include the effects of temperature [11]. The dependence of the displacement barriers on temperature suggests that graphene should be more easily damaged at higher temperatures. Here, we performed a series of experiments to investigate the effect of temperature and instead reveal a pronounced increase in radiation resistance to the e-beam with increasing temperature. This apparent contradiction arises because of adatom surface diffusion and self-healing, as well as the rapid diffusion of point defects away from the area of generation. Combined, these effects result in a net increase in the radiation tolerance of graphene under localized e-beam exposure.

A $100 \mathrm{kV}$ e-beam in a Nion UltraSTEM U200 was used to mill arrays of holes in graphene from room temperature up to $1000{ }^{\circ} \mathrm{C}$, using a Protochips Fusion heater chip to vary the sample temperature. A medium angle annular dark field (MAADF) image of an example array of holes milled at $800{ }^{\circ} \mathrm{C}$ is shown in Figure 1a. Each location in this $5 \times 5$ array was successfully milled, however we can see that several holes healed by the time the final image was acquired. This is due to $\mathrm{C}$ adatom and hydrocarbon diffusion along the graphene surface which acts to transport material to the undercoordinated atoms at the hole edges. The edges then bond to the adatoms and repair the holes [12]. Between the mill locations we can see strings of reconstructed vacancies which diffused away from the e-beam during milling. The diffusion of point defects away from the area of generation gives the graphene an effective mechanism to absorb increased damage without apparent deterioration at the mill location. Given the increased radiation resistance, much longer exposures of the graphene were possible at higher temperatures with the $100 \mathrm{kV}$ e-beam. The inset shows an aligned stack of twenty sequential images of the graphene. No apparent damage was observed during the acquisition. Figure $1 \mathrm{~b}$ shows the progression of a hole being milled at room temperature. The upper half shows the detector intensity over time. The lower half shows the repeatedly acquired images of the circular mill location. With this detailed atomic information, we can begin to extract the precise evolution of defect formation at various temperatures.

Similar mechanisms may also apply to other 2D materials, enabling a higher level of radiation tolerance and greater control over e-beam-induced alterations [13]. 

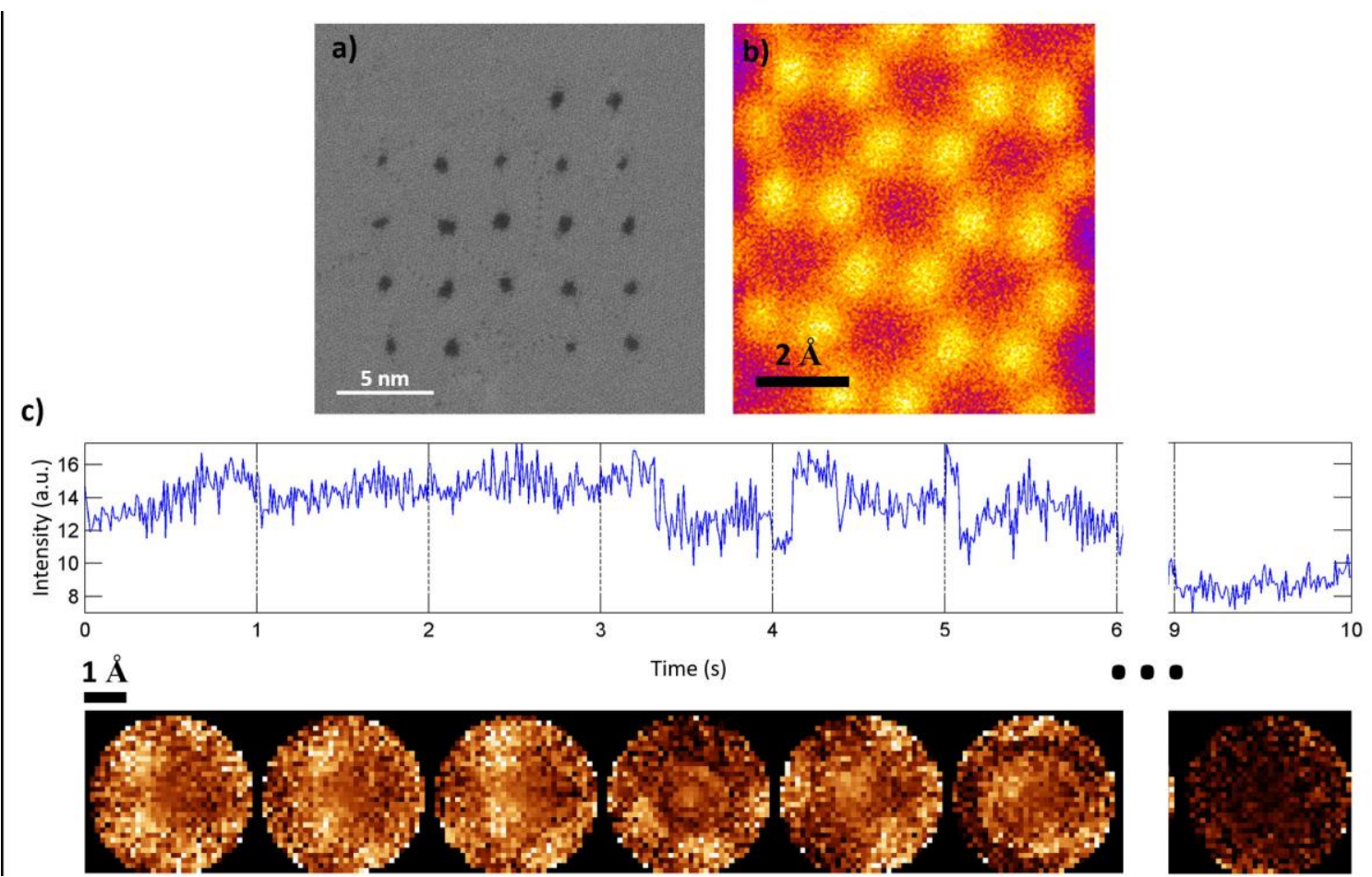

Figure 1. Arrays of holes were milled in graphene with a $100 \mathrm{kV}$ e-beam at various temperatures to reveal the radiation resistance as a function of temperature. a) an example hole array milled at $800{ }^{\circ} \mathrm{C}$. Each hole in the 5X5 array was successful, however, recaptured $\mathrm{C}$ adatoms act to heal the holes after they are milled. b) is an aligned twenty-frame stack acquired at $900{ }^{\circ} \mathrm{C}$. No defects were observed to form during the acquisition. c) Example of the data gathered during the drilling process. Upper panel shows a plot of the detector intensity through time. Lower panel shows atomically resolved images acquired during the drilling process. Each circular scan in this example was acquired over one second, corresponding to the time steps in the upper panel.

\section{References}

[1] R Patrik and T Björn, Nanotechnology 21 (2010), p. 302001.

[2] W Han, et al., Nature Nanotechnology 9 (2014), p. 794.

[3] O Dyck, et al., Nature Reviews Materials 4 (2019), p. 497-507.

[4] SV Kalinin, A Borisevich and S Jesse, Nature 539 (2016), p. 485-487.

[5] M Tripathi, et al., Nano Letters 18 (2018), p. 5319-5323.

[6] T Susi, JC Meyer and J Kotakoski, Ultramicroscopy 180 (2017), p. 163-172.

[7] T Susi, et al., Physical Review Letters 113 (2014), p. 115501.

[8] O Dyck, et al., Carbon (2020).

[9] O Dyck, et al., Small 14 (2018), p. 1801771.

[10] O Dyck, et al., Applied Physics Letters 111 (2017), p. 113104.

[11] AI Chirita Mihaila, T Susi and J Kotakoski, Scientific Reports 9 (2019), p. 12981.

[12] R Zan, et al., Nano Letters 12 (2012), p. 3936-3940.

[13] This work was supported by the U.S. Department of Energy, Office of Science, Basic Energy Sciences, Materials Science and Engineering Division and was performed at the Oak Ridge National Laboratory's Center for Nanophase Materials Sciences (CNMS), a U.S. Department of Energy, Office of Science User Facility. 